\title{
Preuve et épreuves en sciences sociales : pour une sociologie négative
}

Pierre Livet

\section{(e) OpenEdition}

Journals

Édition électronique

URL : http://journals.openedition.org/ress/382

DOI : $10.4000 /$ ress.382

ISSN : 1663-4446

Éditeur

Librairie Droz

Édition imprimée

Date de publication : 10 décembre 2003

Pagination : 75-92

ISBN : 2-600-00913-2

ISSN : 0048-8046

Référence électronique

Pierre Livet, «Preuve et épreuves en sciences sociales : pour une sociologie négative », Revue européenne des sciences sociales [En ligne], XLI-128 | 2003, mis en ligne le 11 novembre 2009, consulté le 19 avril 2019. URL : http://journals.openedition.org/ress/382 ; DOI : 10.4000/ress.382 
Pierre LIVET

\section{PREUVE ET ÉPREUVES EN SCIENCES SOCIALES : POUR UNE SOCIOLOGIE NÉGATIVE}

\section{PREUVE OU ARGUMENTATION?}

Les sciences sociales sont elles dépourvues de modalités de preuve? On peut espérer prouver de deux manières, soit par une déduction théorique, soit par une expérimentation, appuyée elle aussi sur une déduction. Le problème des sciences sociales est alors d'une part que les phénomènes qu'elles étudient sont historiques et ne sont donc pas répétables, ce qui interdit une procédure expérimentale classique, d'autre part que les déductions théoriques, pour être démonstratives, exigeraient une formalisation poussée. Or même dans la science sociale la plus formalisée, l'économie, la formalisation est silencieuse sur les processus effectifs qui déterminent les choix des agents. Une des raisons en est que, dès que l'on tente de modéliser ces processus, par exemple de modéliser l'effet des représentations des agents sur leurs actions et les effets collectifs de ces actions, on aboutit à remplacer l'idée de vérité par celle de théorie auto-réalisatrice (la théorie qui fournit les représentations qui sont confortées par les effets collectifs des actions résultant de ces représentations est auto-réalisatrice). Mais il y a une multiplicité très grande de théories auto-réalisatrices, si bien que nous n'avons pas le moyen de savoir dans laquelle nous nous trouvons ${ }^{1}$. C'est la difficulté inverse de la non répétabilité: la prolifération des modèles compatibles avec les données. Le chercheur en sciences sociales peut chercher refuge dans l'analyse des corrélations statistiques. Cependant, là aussi il y a indétermination, dans la mesure ou ces corrélations sont dépendantes des catégories choisies pour les statistiques. Et une fois une corrélation établie, il reste à l'expliquer, ce qui nous ramène aux difficultés précédentes.

Il semble alors raisonnable d'abandonner l'idée de preuve et de se replier sur celle d'argumentation. Une argumentation est valide seulement dans un contexte d'objets donnés, si l'on y considère comme normales et admises un certain nombre d'inférences usuelles, et sous réserve qu'on ne rencontre pas de contreargument qui soit sans réplique. Toute argumentation est donc relative et provisoire, mais tant que nous n'avons pas mieux, nous devons nous en contenter. Davantage, le fait qu'un argument puisse résister à des contre-arguments, et rester stable dans cet océan d'instabilité, lui donne une vertu qu'il faut apprécier en raison de sa rareté.

Cf. "Vérités et représentations sociales», intervention de R. Guesnerie au colloque «La vérité dans les sciences ». 
Mais ne peut-on aller plus loin? On peut tout d'abord remarquer que toute argumentation met en place un certain processus de preuve. Il consiste d'une part en une suite d'arguments et de contre-arguments, où la défaite d'un contre-argument par un contre-contre-argument peut prétendre restaurer la solidité du premier argument. Cette suite d'argument et de contre-arguments déroule une série de révisions qui signalent qu'une conclusion initiale n'est plus valide, et désignent donc un changement de contexte comme responsable de cette perte de validité. Le retour à la validité du premier argument signifie donc que l'on est revenu dans son contexte de validité, qui est donc maintenant mieux défini, en tout cas mieux défini que celui qui serait pertinent pour le contre-argument à présent défait ${ }^{2}$.

\section{LA PREUVE AU SEIN DES ÉPREUVES}

On peut ensuite noter que certaines théories récentes de la preuve, dans son domaine d'excellence, la logique, en particulier la théorie de la preuve construite par Girard dans Locus Solum, proposent de rechercher un test de ce que la preuve est bien une preuve. Cette démarche, qui peut sembler paranoïaque, nous rapproche de notre problème. Dans cette perspective, une preuve n'est une preuve qu'adossée à une série d'anti-preuves - des tentatives de prouver la conclusion contraire - qui doivent abandonner dans la confrontation. C'est l'ensemble des deux séries adossées, qui constituent une structure de propos et de réponses, s'adossant les unes aux autres. La preuve est ainsi dégagée comme résistante par sa série d'anti-preuves, La structure qui teste la preuve comme preuve émerge donc comme structure stable d'un océan de tentatives, et c'est sa stabilité qui donne son statut à la preuve.

Ce que nous apprend la théorie de la preuve que nous trouvons par exemple dans Locus Solum, c'est que les preuves ne sont pas des échafaudages que l'on pourrait rejeter une fois le bâtiment construit, que les preuves ne restent des preuves que si elles sont en interaction avec des anti-preuves, des épreuves. Dès lors, d'une part, et l'on peut considérer cela comme une mauvaise nouvelle, toute «preuve» a un régime spécifique qui dépend de son régime d'épreuve, et nous ne pouvons donc pas détacher les constructions théoriques de leur appareil de preuve et d'épreuve. D'autre part, et cela peut être une bonne nouvelle, cette interaction entre preuve et épreuve peut être assurée non seulement dans des sciences qui sont pleinement formalisées, mais dans des disciplines qui le sont beaucoup moins. Il

Ces défaites sont relatives. C'est d'abord au contre-argument de se révéler plus fort que l'argument que nous tenions pour normalement valide. Il peut le faire en exhibant un contexte bien spécifique et mieux défini que le contexte plus vague de l'argument initial, ou bien en montrant que l'argument conduit par inférences à mettre en cause une normalité encore plus couramment admise. Le contre-contre-argument doit aussi se montrer plus fort, et dès lors il peut soit exhiber un contexte encore plus spécifique, mais il risque de n'opposer d'une exception locale, soit plus large, mais alors il doit mieux le définir. Il peut aussi recourir à la deuxième stratégie. On comprend pourquoi, quand le contre-contre-argument réinstalle l'argument, le contexte de celuici soit mieux défini qu'au départ. 
suffit de tenir compte du régime spécifique de relation entre preuve et épreuve qui est celui de ces disciplines.

Mais, même une fois ajoutée cet entourage d'épreuves, qui pourrait nous rapprocher des sciences sociales, la différence entre le logicien et le «social scientist » reste cependant énorme: le premier est capable de définir des règles qui vont isoler cette stabilité, alors que le chercheur en sciences sociales ne peut pas y prétendre.

D'où une idée simple: est-ce qu'en sciences sociales, on ne devrait pas d'abord s'attacher à définir les échecs de preuve, les défaites de l'argumentation? Alors que le logicien construit une structure d'épreuves (la dualité entre preuve et anti-preuves) qui fait émerger une preuve, le chercheur en sciences sociales pourrait bien construire aussi une structure d'épreuves, mais qui fasse émerger les ruptures d'argumentation, autrement dit les ruptures de contexte social. On peut aller plus loin: comme pour chacun de ces contextes, on utilise des inférences usuelles qui suggèrent des scénarios normaux, qui impliquent des normalités, le chercheur en sciences sociales aurait pour tâche de repérer les ruptures de normalité qui peuvent indiquer des changements de contexte. Ce que construirait le chercheur en sciences sociales, ce ne serait pas une structure stable de preuve, mais une structure stable de défaiseurs de preuve, d'où l'idée d'une sociologie «négative».

Mais pourquoi une telle structure serait-elle stable, au lieu d'être simplement totalement chaotique? D'abord parce que les acteurs sociaux n'acceptent pas n'importe quel défaiseur de leurs attentes normales. Il faut encore que ces défaiseurs soient socialement reconnus, ce qui implique une stabilité minimale (dans le groupe social qui acceptent ces normalités, et qui est sensible à des défaiseurs). Ensuite parce que les défaiseurs permettent à un groupe social de se différencier des autres groupes sociaux - ceux qu'il ne veut plus être, et ceux qui sont ses compléments dans la structure sociale. Les défaiseurs ayant pour office de dresser des frontières, il faut que ces frontières soient reconnues par les deux groupes, ce qui les stabilise encore davantage. Enfin parce que, même et précisément quand les acteurs appartiennent à la fois à plusieurs groupes, et changent donc euxmêmes souvent de contexte, ils doivent disposer de procédés explicites ou implicites pour laisser tomber certaines attentes normales dans un premier contexte et en endosser d'autres normales dans un autre contexte. Pour un même acteur, ces changements de contexte sont répétés souvent (de la maison au bureau, du bureau à l'association, ou aux relations de loisirs, etc.), si bien que les processus de ces changements doivent être routinisés.

Il reste vrai qu'un changement social de quelque ampleur implique aussi une transformation de ces modes routiniers de changement de normalités entre contextes sociaux. Les stabilités sociales ne sont pas définitives. Mais cela n'est pas gênant: si la méthode en sciences sociales consiste à repérer les ruptures de contextes, il suffit que ces ruptures ne soient pas de tous les instants, et qu'elles restent routinières pendant un certain temps, pour que l'on puisse aussi repérer de manière fiable les transformations importantes de ces modalités de changement de contexte. En revanche, il n'est pas évident que l'on puisse isoler un système de contextes et de ruptures de contextes qui soit entièrement valide sur une période donnée, puisque très probablement, les changements de ces routines de passage 
d'un contexte à un autre ne se produisent pas tous ensemble, mais de manière décalée.

Concevoir les faits sociaux comme ces changements de contextes (ou ces changements des modalités de changement de contexte) qui défont des attentes normales pour en refaire d'autres, c'est aussi définir un objet des sciences sociales de telle manière qu'elles puissent appliquer leurs analyses à elles-mêmes comme objets sociaux. En effet, les différentes théories et même descriptions en sciences sociales ne sont pas homogènes et résultent de choix d'hypothèses. Or ces hypothèses ne sont jamais toujours satisfaites dans les faits (le paroxysme est atteint avec l'économie théorique). Cela n'implique pas qu'elles ne soient pas valides, mais seulement que le véritable objet des sciences sociales n'est pas l'ensemble de données qui valide une théorie ou le modèle qu'elle propose, mais bien l'ensemble des changements de régime sociaux, dont les révisions nécessaires des théories sont des indicateurs. Il n'y aurait qu'une seule théorie sociale stable: ce serait celle qui permettrait d'articuler les changements de théories sociales selon les contextes sociaux, les périodes, les types d'activités sociales. Une théorie sociale à son plus haut niveau serait formellement une théorie des révisions. Ce serait la seule en effet qui permette de relever les mêmes opérations et les mêmes problèmes dans les activités sociales qui se donnent comme théories et recherches sur le social, et les activités sociales qui ne se donnent pas comme telles.

Dans les deux cas, pour revenir au vocabulaire de la théorie girardienne de la preuve, ce sont des conflits entre tentatives de preuve, donc des «épreuves», qui sont présentés. Dans la vie sociale courante, les tentatives de preuve sont simplement les attentes normales, et les conflits, leurs défaites quand on change de contexte. Dans la recherche en sciences sociales, les tentatives de preuves sont des descriptions interprétées, voire des modèles ou des théories, et les conflits sont leurs défaites réciproques. Pour prendre le cas extrème de l'économie théorique, ces conflits consistent simplement à devoir renoncer à certains axiomes (principe de la chose sûre, indépendance par rapport aux alternatives non pertinentes, par exemple) pour pouvoir intégrer des versions stylisées de processus sociaux récurrents. La vie de la recherche en sciences sociales est donc tissée de tentatives de preuve, mais la vie sociale ordinaire l'est aussi. Mais sa visée n'est pas la preuve, mais la mise en évidence d'épreuves reconnaissables, qui nous permettent de nous départager et de nous repérer socialement. Ce qui est social, ce n'est pas le résultat, la preuve conclue, c'est la construction des épreuves.

Mais cependant, il semble souhaitable que le mode d'épreuve diffère, selon qu'il s'agit des pratiques sociales courantes ou des théories des sciences sociales. On souhaite en effet que des théories et des recherches nous apportent des garanties supplémentaires par rapport aux pratiques sociales courantes. Cela est il possible?

Il faut pour le savoir mieux décrire ce que peut être la construction des épreuves qui mettent en interaction ces tentatives de preuve dans lesquelles consiste la vie sociale. 


\section{LES INTERACTIONS PROCHES ET LOINTAINES}

Supposons que les interactions sociales aient la structure suivante: 1) elles exigent des interactions effectives avec des agents en présence les uns des autres, et ces interactions doivent satisfaire certains réquisits. Le domaine de ces interactions effectives, nous le désignerons comme le domaine du proche 2) Dans le même temps, ces agents accomplissent ces interactions en partie parce qu'ils supposent que d'autres agents (qu'ils ne connaissent pas, et qui sont pour eux anonymes, et d'ailleurs substituables) assurent certaines autres interactions. Mais ils n'ont pas accès à ces interactions, qui sont donc pour eux dans le domaine du lointain. Par exemple, en achetant une baguette de pain, je m'assure que le commerçant me donne bien le type de pain choisi et me rend bien ma monnaie, mais par ailleurs je suppose que le pain a été fait avec les produits corrects, que la monnaie n'est pas fausse, et je fais confiance à d'autres personnes pour tester la satisfaction de ces conditions. Ce type de structure correspond à ce qu'on logique linéaire (toujours dûe à Jean Yves Girard) on appelle une connexion «Par»: on relie les éléments d'un domaine non connu, et qui peuvent même être incohérents entre eux, aux éléments connus d'un autre domaine, si on peut assurer qu'entre l'un ou l'autre de ces couples d'éléments (dans le domaine connu ou dans l'inconnu) il existe bien une cohérence entre les éléments. Ici, la cohérence connue et proche, c'est celle qui concerne la fourniture d'un type de pain et d'une quantité donnée de monnaie, et les cohérences lointaines, ce sont les vérifications sur la farine et sur la monnaie.

Notons que les cohérences lointaines peuvent être de deux sortes: soit il s'agit d'interactions qui pour leurs acteurs sont des interactions proches, et nous les visons comme telles - ainsi, nous supposons qu'un contrôleur contrôle des échantillons de farine. Soit il s'agit de cohérences qui reste lointaines pour tout un chacun. Ainsi, le contrôle des échantillons de farine ne garantit pas - loin de là que cette mesure de farine précise qui a servi à fabriquer mon pain a été effectivement contrôlée. Le contrôle de la qualité de farine est alors symbolique, en ce que l'on suppose que la qualité assurée par le contrôle sur une mesure de farine donnée se diffuse aux autres quantités de farines non contrôlées (avec une certaine plausibilité, ce qui suppose une certaine confiance). Ce qui est symbolique ne peut pas être directement contrôlé, mais renvoie indirectement à d'autres contrôles.

Ces interactions sont donc dites cohérentes parce qu'elles sont contrôlées, directement ou indirectement. Il se révèle que la plupart des modes d'interaction sociaux mettent en jeu de tels couples, entre d'une part des routines d'action qui supposent que prima facie les conditions de l'interaction sont satisfaites (les procédures sont supposées correctes par défaut) et d'autre part des procédures qui permettent de mettre à l'épreuve, de temps en temps, ces procédures routinières. Les procédures sociales sont elles-mêmes construites selon la dualité qui fait la preuve, la dualité entre procédure supposées correctes et tests ou mises à l'épreuve de ces procédures.

Il ne faudrait pas en conclure que les acteurs sociaux passent leur temps à tester la validité sociale de leurs routines. Contrairement aux preuves logiques, qui permettent de tester chaque étape de la preuve, les mises à l'épreuve sociales n'ont jamais eu l'ambition de tester toutes les étapes des interactions. Cela est d'ailleurs contraire au mode même de fonctionnement des interactions sociales. Il 
semble reposer sur une structure très largement lacunaire. Si nous reprenons l'exemple de l'achat d'une baguette, le client teste - de temps en temps - le rendu de la monnaie, préfère éviter de prendre une baguette un peu trop brûlée, et, parfois, se rend compte que le prix de la baguette a augmenté. Mais il ne procède à aucun test sur la composition de la farine utilisée, sur la frappe de ses pièces de monnaie, ni sur la fixation du prix de la baguette. Il suppose que ces tests sont faits par d'autres. Et ces autres acteurs sociaux (différents fonctionnaires) supposent évidemment que les boulangers, même quand ils ne sont pas contrôlés, utilisent pour la plupart les farines autorisées, affichent un prix de la baguette conforme au prix du marché, que les clients continuent à vouloir consommer des baguettes, etc. Autrement dit, les interactions sociales comportent toutes une part de test, et une part de confiance dans d'autres tests lointains, confiance qui est le fondement de l'extension «symbolique» des garanties gagés sur des contrôles indirects.

Nous pouvons alors nous intéresser aux modalités de ce contrôle, et surtout aux sensibilités à des distorsions, qui nous amènent à conclure que le contrôle n'est plus assuré. Or nous nous apercevons que ces sensibilités à des «défaiseurs» de l'hypothèse de cohérence contrôlée ne sont pas les mêmes pour les interactions proches et pour les interactions lointaines.

Pour ce qui est de l'interaction proche et de sa cohérence, les choses sont assez simples (la monnaie rendue ne correspond pas à la somme donnée moins le prix de la baguette, etc.). Nous serons donc très vigilants sur ces points. Mais nous serons très tolérant sur d'autres - il est mal vu qu'un commerçant teste chacun de nos billets pour voir s'ils ne sont pas faux, et inversement nous ne testons pas la validité des pièces qu'il nous rend. Pour les interactions supposées lointaines et anonymes, il faut repérer quels sont les signes qui déclenchent des pertes de confiances dans ces interactions (une perte de confiance dans la qualité du pain, qui est suspecté de fabrication industrielle, une perte de confiance dans la qualité de la monnaie, etc.). Nous pouvons donc faire confiance de manière assez aveugle pendant très longtemps, sans ouvrir la moindre enquête sur la cohérence des interactions lointaines, mais si l'on nous donne des informations qui nous rendent l'un de ces interactions suspectes, cela peut très vite s'étendre à d'autres (nous sommes persuadés que tous les maires qui disposent d'un parc immobilier en font profiter les membres influents de leur parti).

Comment expliquer cette combinaison, dans une interaction locale, d'une plus grande sensibilité, mais aussi d'une grande capacité d'ajustement, de tolérance, qui permet de poursuivre l'interaction, y compris sans être bien certain que la démarche adoptée suit bien «la règle» supposée associée à l'interaction? Par le fait que les acteurs peuvent et doivent ajuster leurs révisions réciproques les uns aux autres en temps réel. Inversement, pour ce qui est des tests lointains supposés, les acteurs sociaux font confiance, mais ils ne disposent pas de capacité d'ajustement en temps réel puisqu'ils ne sont pas en charge de l'interaction: dès qu'un dysfonctionnement est révélé, leur seul ajustement consiste soit à continuer à faire confiance malgré tout, soit à sombrer dans la perte de confiance et donc à entamer la dégradation de l'institution sociale.

Il semble donc plausible de soutenir que les interactions sociales « ordinaires » établissent bien des interactions entre interactions proches et interactions lointaines, au besoin en supposant les interactions lointaines établies tant qu'on n'a pas la preuve du contraire. Les relations sociales sont donc insécables, mais 
admettent une certaine distension. Et c'est là d'ailleurs le comportement du connecteur «Par» dans une recherche de preuve. Partant d'une conclusion, on remonte vers les axiomes en éliminant le connecteur, mais il n'est pas nécessaire de développer au même niveau de cette remontée les composants des deux formules reliées. Il suffit de développer les composants d'une des formules. On pourra développer les composants de l'autre formule à une étape de la remontée vers les axiomes aussi tardive qu'il sera possible.

\section{LES SCIENCES SOCIALES SONT ELLES PLUS EXIGEANTES QUE LES FAITS SOCIAUX?}

La question est alors: les théories sociales (ici les théories sociologiques) peuvent-elles construire un mode de mise à l'épreuve qui ait une structure plus forte et qui assure des liaisons plus exigeantes que celle de notre connecteur «Par», qui se borne à gager une interaction lointaine sur une interaction locale, ou bien une interaction locale sur une interaction lointaine?

La structure logique la plus exigeante en ce domaine, c'est celle du «Fois», qui exige, pour relier deux domaines par une cohérence transversale, qu'existe déjà dans chacun de ces domaines une cohérence, et qui exige même, dans la recherche de preuve et sa remontée, de traiter les cohérences en question ensemble, au même niveau de remontée, de mener leur analyse de manière corrélée à chaque étape.

Il semble bien que ce soit l'idéal des sociologues. En effet, supposons qu'ils aient affaire à une connexion «Par». Les acteurs, eux, font confiance à une cohérence lointaine pour gager sur elle la possibilité de leur interaction locale. Ils ont des modes de révision distincts pour le lointain et pour le proche. Mais le sociologue qui observe cette disparité accomplit précisément ce que ne fait pas la connexion «Par» de la vie ordinaire. Il fait une enquête sur la réalité de la cohérence lointaine, que ne peuvent pas faire les acteurs sociaux, et sur la dépendance de l'interaction locale par rapport aux représentations de cette cohérence lointaine. Inversement, il analyse la dépendance de l'interaction lointaine par rapport aux différentes interactions locales. Ce faisant, il remarque comment les processus de révision des acteurs fonctionnent de manière différente pour le proche et le lointain. Il remarque aussi ce que peuvent être les effets en chaîne des interactions locales, jusqu'à produire des effets collectifs qui sont en contradiction avec l'interaction lointaine supposée - effets que peut masquer la persistance de la confiance dans cette interaction lointaine. Il montre donc à la fois comment fonctionne notre connexion «Par», à quels dysfonctionnements elle est sensible, et comment elle peut masquer d'autres dysfonctionnements.

Le travail des sociologues prend donc un sens plus limité mais aussi plus précis si l'on considère qu'il se greffe sur cette structure de liaisons lacunaires entre interactions locales dont les acteurs présents sont en charge, et interactions lointaines qu'ils présupposent opérées. D'une part, les enquêtes et les descriptions des sociologues ont pour effet essentiel de permettre de «rapprocher» des interactions qui étaient lointaines, et donc de mettre en évidence des ajustements locaux qui sont différents de ce qu'on en pensait quand on les voyait comme des 
interactions lointaines. D'autre part, les études statistiques suggèrent des liens entre des interactions lointaines, liens qui restaient inaperçus des acteurs; elles accentuent alors leur sensibilité aux conditions limites de ces interactions considérées comme lointaines. Bref les études des sociologues introduisent dans l'étude des interactions lointaines des opérations qui étaient réservées aux ajustements locaux, et rendent aussi plus sensibles à leurs dysfonctionnements. En quelque sorte, les études sociologiques rapprochent les unes des autres les conditions de test d'interactions sociales éloignées les unes des autres.

L'enquête du sociologue a donc pour fin et pour résultat de lui montrer dans quelle mesure la connexion «Par», la relation sociale entre le proche et le lointain, est en déficit sur une connexion «Fois». Autrement dit, il nous révèle dans quelle mesure la structure d'épreuves mise en place par la connexion sociale «Par» diffère d'une structure d'épreuves plus complète et moins lâche. Mais il ne faudrait pas en conclure que la théorie du sociologue «complète» et « resserre » la structure d'épreuves sociales au point de satisfaire toutes les contraintes d'une connexion «Fois». On reste dans le domaine du social, que nous avons défini comme un domaine de tentatives de preuves, qui nous fournissent des repères sociaux bien davantage par les défaites réciproques de ces tentatives que par leurs réussites, ou, plus généralement, par les conflits entre ces tentatives de preuves. Le social est le domaine des épreuves qui n'aboutissent pas à des preuves.

On peut se demander si ce n'est pas là un domaine bien trop mal défini pour donner lieu à une exploration qui présente quelque intérêt et surtout quelque cumulativité dans ses résultats. Revenons sur notre analogie avec le test de preuve dans la construction de Girard. On comprend que la preuve puisse être en quelque sorte sélectionnée comme ce chemin de démonstration qui est laissé intact par les abandons successifs des tentatives d'anti-preuves avortées. Mais il semble impossible d'obtenir une sélection similaire parmi les tentatives d'anti-preuves. Cependant, considérons le rôle que nous avons attribué au travail des sociologues. Ils travaillent dans un domaine où tout est tentative de preuve, et où le plus probable est qu'aucune tentative n'arrivera à dominer définitivement les autres ${ }^{3}$. Le sociologue, nous l'avons dit, rapproche des interactions qui chacune fonctionnent sur des normalités supposées valides (mais dont on sait d'avance qu'elles ne seront pas valides dans tous les cas). Autrement dit, il attise des conflits qui n'apparaîtraient peut-être pas aussi rapidement sans son travail.

Qu'est ce qui peut être alors sélectionné par ces conflits entre tentatives de preuve? Aucune tentative de preuve, assurément. En revanche, les structures de révision qui articulent ces conflits sont sélectionnées. Les réseaux d'arguments et de contre-arguments se simplifient peu à peu au fur et à mesure que les contre-

Une sociologie cognitive pourrait espérer identifier des mécanismes cognitifs dédiés au social, et qui soient identiques à travers toutes les sociétés. Très probablement, il s'agira de mécanismes qui assurent la convergence, et d'autres qui accentuent les divergences. Il s'agira aussi de mécanismes qui permettent de distinguer des contextes et des amodes d'activité appropriés pour chacun d'eux, donc de distinguer les domaine restreints de validité des attentes normales (nos «tentatives de preuve»), et de réviser ces attentes quand on change de contexte. Mais dès qu'on entre dans des sociétés évolutives, on sait d'avance que l'essentiel des conduites sociales est sujet à des transformations plus ou moins rapides, si bien que les tentatives de preuve du passé rencontreront des défaiseurs dans l'avenir. 
$\ldots \mathrm{n}+1$--arguments défont des contre-...n-arguments et réinstallent donc des arguments-....n-1. Cependant cette simplification ne peut prétendre dégager un noyau définitivement stable d'arguments, car de nouvelles informations arrivent et enrichissent nos catégories, si bien que l'ensemble des arguments s'étend alors que l'ensemble des contre-arguments se réduit. Ce qui est donc peu à peu sélectionné par ces conflits entre tentatives de preuves, ce ne sont ni des preuves, ni même des tentatives de preuves stables pour un temps, mais seulement des systèmes de réduction des chaînes de contre-arguments ${ }^{4}$ et les bifurcations de ces chaînes.

Ces réductions vont avoir deux effets. Le premier est de réduire l'extension, que nous dirons verticale, de la chaîne d'arguments et contre-arguments, puisque le contre-contre-argument, s'il ne nous permet pas vraiment d'éliminer le contreargument, nous permet au moins de savoir que, moyennant la validité de ce contre-contre-argument, nous pouvons revenir dans le contexte initial, qui est d'ailleurs mieux précisé par là. Le second est d'augmenter l'extension, que nous dirons horizontale, des différences entre contextes. Le contre-argument, en effet, peut prétendre rester valide même alors que le contre-contre-argument l'a affaibli, mais c'est au prix a) de reconnaître que le contexte commun à l'argument et au contre-contre-argument est stabilisé (relativement); b) qu'il existe un autre contexte auquel ce contre-argument a fait appel. Ce dernier contexte n'est pas encore stabilisé, il le sera quand le contre-argument aura lui-même montré qu'il peut résister à un autre contre-contre-argument grâce à un contre-argument de $3^{\text {eme }}$ rang qui stabilise son contexte, etc. Cette structure qui réduit les chaînes, stabilise les contextes, et les différencie, reste stable indéfiniment, puisque même si on ajoute un contre-argument qui défait l'argument réinstallé par de précédentes réductions, ces réductions, elles, ont été utilisées pour rendre cette défaite plus rapide et plus accessible, et pour donner une stabilité relative à ces contextes et les différencier. Tout ce qu'ajouteront les nouveaux arguments et contre-arguments, ce sont justement des extensions de ce système de réductions et de contextualisations, mais des extensions conservatrices ${ }^{5}$.

Le travail du sociologue ne consiste donc pas à valider une tentative de preuve parmi d'autres. Il consiste à construire le réseau de réduction et de contextualisation des contre-arguments, donc de réduction des conflits entre tentatives de preuves, ou dans un vocabulaire plus proche du social, entre normalités sociales.

${ }^{4} \quad$ N'est ce pas trop exiger? Il serait possible que tel contre-contre-argument, supposé réinstaller l'argument initial, et donc nous éviter le détour par le contre-argument, soit contesté par un contrecontre-contre-argument qui se trouve en même temps donner un sens nouveau au contre-argument. Mais d'une part, ce nouveau pas va instaurer un nouveau court-circuit (permettant de ne pas faire le détour par le contre-contre-argument) et d'autre part, une telle situation exige que l'on ait changé le sens du contre-argument, qu'on ait changé de contexte. On peut représenter cela par une sorte de bifurcation. Le premier court-circuit reste donc valide avant la bifurcation, et c'est pour la série qui commence avec la version bifurcante du contre-argument que le nouveau court-circuit est valide. Il est donc toujours possible de développer par extension - sans suppression des court-circuits précédents - l'arbre qui résulte des réductions de circuits argumentatifs.

s Nous tenons là un moyen de différencier des cultures sociales: dans deux cultures différentes, on ne recours pas aux mêmes chaînes refermées d'argument, contre-argument, contre-contre-argument. On ne stabilise donc pas et on ne différencier donc pas les contextes de la même manière du moins pour un sous-ensemble important de contextes. 
Une fois qu'un contexte est ainsi supposé stabilisé par les acteurs -qui n'attendent pas le sociologue pour se lancer dans ces conflits de tentatives de preuves et tenter certaines réductions et contextualisation - il peut servir de cohérence symbolique ou lointaine: il sert alors d'interaction lointaine sur laquelle des interactions proches peuvent se construire. Quand le sociologue compare et relie les modes de contrôle des interactions lointaines et ceux des interactions proches, soit il va simplement tenter d'expliciter les modes de réduction et de contextualisation qui organisent les contextes de ces interactions entre le lointain et le proche (c'est la tentative de Boltanski et Thévenot dans un premier temp). Soit il va être amené à montrer que le rapport entre le lointain et le proche ne fonctionne pas comme supposé - c'est le propos de Bourdieu et Passeron, puis, sur un tout autre mode, de Dubet. On observe alors le phénomène suivant: le contexte précédent fonctionne toujours cahin-caha, mais il est soupçonné, les acteurs sont plus exigeants sur ses contrôles; d'autres contextes se mettent peu à peu en place. Ainsi, une fois le rôle de l'école comme facteur d'égalité dénoncé, d'une part il reste posé, mais avec des exigences plus grandes, d'autre part se constituent d'un côté des stratégies opportunistes d'utilisation des méandres du système scolaire - nécessitant une différenciation plus fine des contextes et reliées à une symbolique de rationalisation et de compétition - de l'autre des réactions de refus d'un système coûteux et sans perspective - pour les jeunes des banlieues, qui tentent de se créer des contextes sociaux transitoires (parce qu'ils fonctionnent surtout sur des interactions proches, sans arriver à se stabiliser sur des interactions lointaines, parce qu'ils ne disposent que de contre-arguments qui se détruisent les uns les autres).

En résumé, les figures contextuelles autre fois stabilisées restent reconnues comme telles. Mais se développent à côté d'elles et aussi en conflit avec elles d'autres tentatives de contextualisation, qui soit réussissent à réduire leur chaîne de mises en question pour former un contexte plus stable socialement, qui peut donc restaurer un interaction entre le proche et le lointain (le modèle stratégiquecompétitif), soit ne réussissent pas encore à réduire leur chaîne de mises en question, et qui ne forment un contexte repérable que par la similarité de leurs interactions proches, sans référence à des interactions lointaines (d'où la perplexité du sociologue devant «la galère»!). Or ce sont là les formes que peuvent prendre les tentatives de preuve: des anti-preuves qui se réduisent les unes les autres en stabilisant et différenciant des contextes, cette stabilité et différenciation étant pleinement activée (quand le modèle social est supposé fonctionner) ou bien partiellement désactivée (quand on se focalise sur d'autres contextes), ou bien des anti-preuves qui n'arrivent pas encore à réduire leurs chaînes de défaiseurs et qui ne peuvent donc qu'emprunter leur minimum de stabilité à des situations parasites par rapport à d'autres contextes plus stabilisés (familles, par exemple). Si nous nommons de manière générique tous ces conflits entre tentatives de preuves des tentatives de révision, la tâche du sociologue est de montrer la structure des tentatives de révision, et dans quelle mesure ou non elles isolent des stabilités locales et différencient des contextes. 


\section{LES PARADOXES MÉTHODOLOGIQUES DE QUELQUES THÉORIES SOCIOLOGIQUES}

Mais le problème de bien des études sociologiques, c'est qu'elles présentent ce travail d'élucidation des modes de stabilité des contextes, de leur activation ou désactivation, et des conflits qui parviennent ou non à réduction, soit comme une reconstitution des raisonnements des agents, dont les interactions produisent les effets collectifs observés (individualisme méthodologique), soit comme un dévoilement de fonctionnements sous-jacents aux fonctionnements institutionnels affichés (école de Bourdieu). Autrement dit, alors que les interactions sociales fonctionnent comme des mises à l'épreuve très locales sur fond de confiance en des interactions lointaines, et que les travaux sociologiques ne font que resserrer le tissu de ces mises à l'épreuve, mais en devant toujours présupposer et que les tests restent locaux, et que le réseau d'interactions sociales reste lacunaire et hypothétique, la plupart des études sociologiques (des théories sociales) présentent leurs «preuves» comme de simples outils pour valider un tableau des fonctions et relations sociales supposé complet et stable, et proposé par leur théorie particulière. Or un tableau se lit sur le mode du «Fois », en traitant en même temps toutes ses données, ou du moins en tenant compte à tout étape de l'analyse de l'ensemble de ses relations. Il ne correspond donc en rien à cette structure lacunaire du «Par», envoyant des ponts sur des abîmes, faisant dépendre une interaction concrète de la représentation abstraite ou symbolique d'une interaction actuellement inaccessible, et encore moins à la structure partiellement différenciée et partiellement chaotique des tentatives de preuve.

La théorie de Bourdieu n'est pas loin du compte, puisqu'elle prétend nous révéler l'envers des représentations sociales considérées comme normales, en montrant à quelles stratégies implicites elles obéissent. Ces stratégies combinent deux modalités, celle de l'habitus, qui ne peut en fait fonctionner que dans des interactions concrètes, et celle du capital symbolique, qui fait fonctionner des interactions lointaines hypothétiques. Mais il semble que Bourdieu ait inversé les facteurs. Son travail de sociologue consistait bien à révéler dans quelle mesure le rapport entre interactions locales et interactions lointaines reposait sur des faux semblants. Mais ce qu'il nous propose comme envers de ces faux semblants, c'est le composé entre l'habitus et le capital symbolique, donc entre une notion qui est parfaitement adaptée à l'étude des interactions locales, l'habitus, et une notion réservée aux interactions lointaines et symboliques, le capital symbolique. En effet, l'habitus prend sens quand on observe des interactions effectives dont on peut expliciter les présupposés comme étant des règles et les attentes normales. Le capital symbolique au contraire consiste en des représentations non directement vérifiées, mais dans lesquelles les autres partenaires vont avoir confiance, et qui assurent aux acteurs une réputation qui franchit les frontières de leurs interactions locales (cf. Russell Hardin, pour une autre analyse de cette notion de réputation).

Mais Bourdieu ne s'aperçoit pas de la dualité de statut de ces deux notions, il les suppose compatibles sans plus. Il fait comme si la notion d'habitus pouvait s'appliquer à des modes de confiance gagés sur des symboles, pour des interactions lointaines. Mais on est alors incapable de préciser le contenu de l'habitus (quelles règles et attentes normales guident une stratégie de maintien ou d'accroissement du capital symbolique, cela n'est jamais épelé). 
Bourdieu a donc procédé à une dénonciation qui est bien du rôle du sociologue, mais il nous propose une structure qui est celle des interactions sociales non dénoncées, et il nous la propose comme la véritable interaction sociale, cet envers caché qui permet de dénoncer les apparences. Il a confondu les genres. Il aurait au contraire dû reconnaître la dépendance des interactions locales (le choix de telle filière dans telle école, le statut social des parents dans telle bourgade) et des interactions lointaines (la possibilité d'ascension sociale, l'accès à un savoir commun), et dès lors il pouvait interpréter les statistiques comme il l'a fait avec Passeron, en notant que la corrélation restait forte entre filière courte et statut social faible, donc en mettant en cause la cohérence de l'interaction sociale lointaine. Il n'avait alors nul besoin de supposer un mécanisme caché jouant entre habitus et capital symbolique. Son erreur est de n'avoir pas saisi que les interactions sociales ne sont pas complètes ni exemptes de défaiseurs. Dès lors elles reposent sur des hypothèses risquées et non totalement vérifiées sur les interactions lointaines. Mais les effets collectifs qui résultent des interactions locales qui, elles, sont mieux contrôlées (mais toujours ouvertes à des défaiseurs, puisqu'elles prennent le risque de la tolérance) peuvent se révéler en conflit avec ces hypothèses. Révéler ce conflit est bien du devoir du sociologue, mais il n'a pas pour autant à supposer que cette révélation lèverait un voile et remplacerait une vision erronée, une représentation complète mais illusoire, par un mécanisme valide et tout aussi complet. Car alors c'est lui qui tombe dans l'illusion de la complétude de la représentation sociale et de son action.

Qu'en est-il de la théorie concurrente de celle de Bourdieu, celle de Boudon? Elle partait d'abord d'une analyse statistique des corrélations, en se demandant si la variance de tel facteur était en corrélation avec la variance de tel autre, et en éliminant les variances qui n'étaient pas en corrélation. Puis elle proposait une reconstruction d'un raisonnement des acteurs, supposés à la recherche de la maximisation de leur utilité sous contraintes. C'est ainsi que Boudon pensait avoir montré que la variance des revenus suffisait à expliquer la variance des temps d'éducation, en supposant que pour les parents plus pauvres le sacrifice lié à la poursuite des études était plus grand, et la probabilité de son efficacité plus faible. Plus tard, Boudon a ajouté au raisonnement en termes de maximisation de l'espérance d'utilité un raisonnement en termes de «bonnes raisons », qui pouvait intégrer des valeurs reconnues socialement. Il faut voir que la reconstruction rationnelle en termes d'espérance d'utilité est toujours sous-déterminée. Plusieurs raisonnements pourraient expliquer les corrélations entre variances constatées, puisque les choix des agents dépendent à la fois de leurs préférences et de leurs croyances, et que l'on ne sait pas clairement comment séparer les unes des autres. Même si ce problème était résolu, il est toujours possible d'imaginer à un agent les préférences que l'on veut. Ce qui contraint le modèle, c'est seulement le souci de rendre les choix des agents cohérents entre eux. Mais puisqu'on peut modifier les préférences et les croyances, il existe toujours beaucoup de manières de restaurer la cohérence. Pour le sociologue, on peut ajouter une contrainte supplémentaire: il faut que les préférences ou valeurs que l'on prête aux agents soient largement répandues dans les représentations de la population considérée. Or comment peuton s'assurer non seulement de la possibilité que ces représentations soient partagées, mais du fait qu'elles le soient, ou encore, comment peut-on s'assurer que la cohérence supposée présente une forte plausibilité d'être similaire à celle suivie 
par les agents? C'est là que l'on constate un vide dans la méthodologie boudonienne. Rien ne nous est dit sur ce point. Or les réponses ne manquent pas. Pour tester la cohérence, il faut tester les réactions de rejet d'incohérences. Pour tester le partage des représentations, il ne faut pas se borner à les recenser dans des entretiens -c'est déjà un travail indispensable-, mais il faut tester le refus de partage de représentations légèrement différentes. Autrement dit, il faut tester les motifs de révision qui animent les acteurs face à ce qu'ils ressentent comme incohérence et ce qu'ils rejettent comme anti-valeurs. Et pour tester ces motifs de révision et ces rejets, il faut prendre garde aux différences dans les sensibilités aux distorsions, différences qui se manifestent entre les interactions proches et les interactions lointaines.

Or l'individualisme méthodologique tel que Boudon l'a développé ne tient pas compte de cette différence. La théorie du choix rationnel non plus. Plus exactement, la théorie du choix rationnel fait primer la micro-économie sur les considérations macro, et donc elle prétend considérer des intérêts personnels des individus, ceux là mêmes qui s'investissent dans les interactions proches. Mais elle soutient que cela suffit, et qu'on peut expliquer les interactions macro par agrégation des interactions micro. Mais pour cela, il faut aussi pouvoir considérer comme homogène le fonctionnement des raisonnements qui portent sur le proche et de ceux qui portent sur le lointain. Autrement dit, la théorie du choix rationnel fait comme si on pouvait compter sur cette homogénéité. Mais cette homogénéité est un des aspects de l'attitude sociale naïve, qui suppose que ce sont bien les valeurs des interactions lointaines qui inspirent les actions locales tout en s'enracinant en elles.

Or Boudon a passé son temps à mettre en question cette homogénéité! Tout d'abord, en mettant l'accent après Olson sur les effets collectifs d'interactions raisonnables à l'échelle de l'interaction proche, mais désastreux ou non souhaités à l'échelle de l'interaction lointaine. Et cela lui était permis par l'utilisation d'instruments d'agrégation inspirés de l'économie. Ce faisant, il accomplissait son travail de sociologue: il rapprochait les interactions proches et les interactions lointaines, il vérifiait leur couplage de manière plus stricte, et il montrait que l'agrégation des interactions proches ne menait pas forcément aux interactions lointaines sur lesquelles elles étaient gagées, ou, inversement, dont elles étaient supposées garantir l'effectivité. Ensuite en introduisant des concepts assez vagues de «bonnes raisons » et de « dépendance par rapport au contexte» (avec le slogan: «les choix des acteurs sont rationnels, au contexte près»). En travaillant davantage ces concepts, il se serait rendu compte que les «bonnes raisons » ont précisément le statut des cohérences supposées pour les interactions lointaines (cf. nos raisons de voter) et que la dépendance par rapport au contexte est un complexe qui combine la situation propre aux interactions proches et leur référence aux interactions lointaines ( $\mathrm{si}$ mon voisin va voter, je vais me sentir presque contraint de voter, ce qui tient à l'interaction proche, mais lui peut le faire parce qu'il pense que la poursuite de la démocratie exige des votes en nombre suffisant, ce qui renvoie à une cohérence lointaine). Le second Boudon, celui des bonnes raisons, s'est employé à juste titre à combattre des accusations d'irrationalité portée au nom du choix rationnel contre les comportements sociaux effectifs des acteurs (il n'est pas rationnel, dans cette perspective, de voter, le coût dépassant l'influence de mon vote). Mais il a oublié de développer le parallèle de son enquête sur les effets 
pervers pour ce qui est des raisonnements qui tiennent compte à la fois des valeurs générales et du contexte local. Or là aussi, le sociologue peut nous montrer qu'il y a parfois distorsion entre les contraintes de nos interactions locales et les références à des valeurs collectives.

Ainsi Bourdieu ne nous proposait, comme envers du décor social, que ce même décor, qui prétend relier sans problème interactions proches et interaction lointaines. Et Boudon, après avoir bien montré que les effets d'agrégation brisent cette homogénéité, ou que les valeurs n'induisent pas les mêmes sensibilités aux révisions que les choix rationnels qui supposent cette homogénéité, en revient à admettre comme allant de soi la relation entre interactions proches et interactions lointaines. Cette incapacité à se détacher de l'attitude naïve a cependant sa justification: il n'y a pas d'autre attitude initiale pour construire les interactions sociales que l'attitude naïve! On doit toujours la présupposer pour devenir ensuite attentif à l'hétérogénéité qu'elle masque forcément - puisqu'elle gage des interactions contrôlées sur des interactions non contrôlées.

Des sociologues plus contemporains répètent de manière différente cette situation problématique. Dubet propose des acteurs qui utilisent au jour le jour la dispersion des valeurs, donc qui rabattent les interactions lointaines sur les interactions locales, et qui refusent de se concevoir en porteurs de nouvelles valeurs (contrairement à ce que la sociologie des mouvements sociaux de Touraine voulait leur imposer). Mais ce repli laisse place à une dépendance par rapport à des réseaux religieux, mafieux, qui deviennent d'autres références d'interactions lointaines, ce que la théorie de l'acteur de la galère n'étudie guère. Boltanski et Thévenot sont sensibles aux procédures de dispute sur les qualifications des objets et des conduites, quand elles se trouvent à cheval sur plusieurs « cités ». Ce sont là les procédures de révision dont nous pensons qu'elles font le cœur de l'analyse sociologique. Mais ils supposent que les acteurs se réfèrent à une structure morale, qui pour eux combine des aspects des interactions proches (au niveau des «petits») et des interactions lointaines (au niveau des «grands »). C'est déséquilibrer l'analyse, et comprendre les interactions proches à partir de la référence aux interactions lointaines, alors que rien n'assure de cette dissymétrie. Les ethnométhodologues, de leur côté, sont des spécialistes des interactions proches et de leurs présupposés, et ils ont proposé ce que l'on peut considérer comme des tests des seuils de sensibilité qui déclenchent des révisions (par exemple dans les breaching experiments). Mais soit ils se refusent à étudier en quoi ces révisions pourraient remettre en cause les cohérences supposées par les acteurs entre interactions proches et lointaines. Soit ils supposent un peu vite que les présupposés implicites des interactions proches sont en même temps les règles de base de la société (c'est la tendance d'une sociologie wittgensteinienne). C'est se priver du développement de l'outil qu'ils ont pourtant commencé à utiliser.

\section{LA RECHERCHE EN SCIENCES SOCIALES COMME MISE À L'ÉPREUVE DES TENTATIVES DE PREUVES}

Qu'en est-il alors, finalement, des modalités de «preuve» en sciences sociales? Les preuves formelles y ont encore leur place (il s'agit de modèles 
économiques, la plupart du temps, ou de modèles évolutionnistes avec simulation des évolutions des populations), mais elles ont un autre statut: elles ne prouvent rien concernant les interactions sociales. Elles servent plutôt à repérer de manière probante en quoi les tentatives de preuves qui sont l'essentiel de la vie sociale sont bien seulement des tentatives. Etre une tentative de preuve, cela veut dire: 1) proposer une normalité - permettre des attentes normales, engager une règle implicite, etc. ; 2) susciter une sensibilité à des distorsions qui déclenche des révisions ; 3) s'il y a réduction de la chaîne des arguments et contre-arguments, et donc commencement de constitution d'un contexte différencié, proposer une relation toujours risquée entre cohérence locale et cohérence globale, mais aussi prêter le flanc à des analyses qui mettent en question l'efficace de cette relation. Une vraie preuve serait évidemment une normalité qui n'aurait pas d'exceptions, qui ne serait jamais à réviser, et qui assurerait la cohérence entre chaque étape de la preuve et son développement complet. Cela ne semble pas avoir un statut social. L'étude du chercheur en sciences sociales consiste donc à bien décrire la relation entre local et global, ou proche et lointain, et à tester cette tentative de preuve, en en montrant les failles: effets d'agrégation qui ne respectent pas les cohérences lointaines annoncées, révisions locales qui déplacent les contextes, pertes de confiance dans la stabilité des interactions lointaines.

Le problème qui reste dans cette approche est de savoir comment le chercheur en sciences sociales peut certifier que sa description de la relation entre interactions proches et interactions lointaines est correcte, et comment il peut être sûr qu'il a bien mis au jour des agrégations différentes, des déplacements des contextes, des pertes de confiance qui ne sont pas seulement temporaires. Bref comment peut-il prouver que les tentatives de preuve qu'il nous propose sont bien effectives?

En fait, la difficulté n'est pas de prouver que c'est bien ainsi que fonctionnent les interactions sociales. Cela supposerait que ces interactions ont un fonctionnement univoque et que l'on puisse décrire de manière complète. Or ce n'est pas le cas. Nous pouvons seulement repérer d'une part les modes d'ajustements et de révision, d'autres part les distorsions et les failles de ces interactions. Ce que nous montre l'histoire de la sociologie, c'est au contraire que les sociologues n'arrivent pas à prendre suffisamment de recul par rapport aux interactions telles qu'ils se les représentent - or ces représentations sont elles-mêmes des faits sociaux - pour pouvoir contourner l'attitude naïve. Nous partons tous de représentations à la fois prétentieuses pour ce qui est de leur mise en cohérence et d'une incomplétude facile à établir. Ce que le sociologue a pour tâche, c'est seulement de se donner les outils nécessaires pour repérer ces incomplétudes. L'analyse statistique de discours en est un: les représentations explicites sont elles centrées sur les représentations que nous nous figurons centrales? Les statistiques économiques et sociales en sont un autre. Evidemment, il ne faut pas les prendre comme un tableau de la réalité sociale, puisqu'elles dépendent très largement des catégories de regroupement choisies. Mais là encore, il faut prendre les représentations statistiques pour des représentations semi-naïves, et il faut mettre en évidence les distorsions possibles. Il faut ainsi montrer qu'en changeant quelques frontières entre catégories, on change les corrélations. Et c'est là le travail du sociologue.

Comme on le voit, c'est un travail différentiel. Il ne valide pas des représentations de la réalité sociale, il ne valide ni les représentations naïves ni les tableaux 
statistiques. Mais une fois qu'il a montré les distorsions entre les deux, et les distorsions qui découlent des regroupements statistiques, il a donné la preuve de ces distorsions simplement en montrant que des arguments plausibles qui développent ces représentations sont en conflit avec des arguments plausibles qui développent les données statistiques, et de même pour les biais des regroupements statistiques. Ces arguments sont donc sujets à révision, mais pour les réviser, il faut fournir d'autres contre-arguments du même type, donc il faut simplement enrichir le travail du sociologue (et constituer des contextes différenciés). D'autres mises en révision sont possibles en confrontant les raisonnements effectifs explicités par les agents lors d'entretiens, ou encore leurs choix économiques, avec des modèles économiques du choix rationnel, ou avec des modèles cognitifs qui se repèrent sur des biais cognitifs bien documentés, ou encore avec des modèles d'agrégation, éventuellement des simulations évolutionnistes de populations dont les agents disposent d'une gamme limitée de comportements variant en fonction de paramètres. Là encore, on ne montrera pas que les acteurs sociaux agissent en suivant le choix rationnel, ou les biais cognitifs, ou qu'ils se conforment collectivement aux modèles d'agrégation, ou à telle simulation de population. On montrera seulement que, alors que leurs représentations sont compatibles avec telle stratégie (mettons, en théorie des jeux) ou avec telle distribution de rôles et de fonctions de comportement dans une population, les acteurs s'écartent sur certains points des modèles proposés. Autrement dit, les modèles ne servent pas de représentations même simplifiées de la manière dont les agents sociaux interagissent. Ils nous servent de prolongements sophistiqués de nos inférences usuelles, pour nous donner des arguments et des contre-arguments plus riches, qui mettent plus facilement en évidence les incomplétudes des interactions sociales parce que les inférences qu'ils permettent ne sont pas toutes aussi chargées de connotations idéologiques que les représentations sociales initiales. Le sociologue indique ces conflits et distorsions, c'est son travail négatif, et il esquisse la carte des retours sur arguments qui signalent des stabilisations relatives de contextes et des révisions qui signalent des différenciations de contextes - c'est son travail positif. D'autres peuvent en donner des interprétations plus charitables, qui réduisent les conflits en proposant d'autres conceptions de la rationalité ou d'autres modèles. Ce qui sera alors mis au jour, c'est la possibilité de revenir d'une révision à un simple ajustement. Mais, comme il y a incomplétude, très probablement, en changeant les modèles et les conceptions de la rationalité, on va mettre au jour d'autres distorsions.

La validité des découvertes du sociologue qui documentent les incomplétudes des interactions sociales est donc dépendante des extensions nouvelles de la théorie de la rationalité ou des simulations formelles d'interactions de populations. Ce n'est pas là une dépendance particulièrement dérangeante ou originale: elle se rencontre aussi dans les sciences physiques, ou biologiques, dont les conclusions dépendent des capacités d'intégration et d'extension propres aux modèles issus des théories proposées. Mais la différence est que le sociologue ne croit pas qu'un nouveau modèle va lui apporter un tableau plus précis de la réalité sociale, et qui lui corresponde mieux. Il croit simplement qu'un nouveau modèle peut lui permettre de relativiser les précédentes découvertes des incomplétudes et des distorsions des interactions sociales effectives par rapport à leurs cadres de référence annoncés. Une fois que le sociologue a accepté que sa situation épisté- 
mologique implique une approche différentielle, qui relève les distorsions par rapport à une complétude supposée du guidage des interactions sociales par leurs représentations, l'usage des modèles formels prend de lui-même son rôle correct.

\section{CONCLUSION}

Nous avions évoqué en commençant la thèse de Guesnerie: la vérité en sciences sociales se réduit à la véridicité, qui tient elle-même à ce que la théorie proposée peut vérifier son auto-réalisation par les acteurs sociaux qui y croient. C'est là un point de vue d'économiste, et même de spécialiste des «anticipations rationnelles ». Le point de vue du sociologue est complémentaire: il repère précisément, pour ces représentations dont on peut prima facie penser qu'elles ont une puissance auto-réalisatrice - au sens précis où les acteurs, en croyant à la cohérence lointaine qu'elles assurent, se lancent dans des interactions locales dans lesquelles ils ne s'engageraient pas sans cette croyance - leurs modalités de non auto-réalisation. L'économiste peut démontrer la capacité auto-réalisatrice de son modèle, le sociologue utilise les modèles comme arguments pour mieux mettre à l'épreuve les ponts que font les représentations des acteurs sociaux entre leurs interactions proches et leurs interactions lointaines, et révéler leurs incomplétudes. Les preuves du chercheur en sciences sociales ne sont que des mises à l'épreuve.

Cependant, nous pouvons malgré tout donner raison à Guesnerie. Nous avons dit que le sociologue, en mettant en évidence les incomplétudes, ne sélectionnait pas des tentatives de preuve, ou des normalités sociales qui restent indéfiniment valides. Il sélectionnait en revanche des systèmes de réduction - de simplification, de stabilisation d'un contexte - des conflits entre tentatives de preuves ou entre normalités sociales. Or quand le sociologue a bien fait ce travail, son système de réduction devrait pouvoir rester relativement stable. De nouveaux contre-arguments, une fois le travail du sociologue connu, vont devoir s'intégrer dans sa structure de réduction. Soit ils y sont absorbés et réduits, soit il s'agit véritablement de nouveaux arguments, mais l'extension qu'ils procurent est conservatrice par rapport au système de réductions. Une fois ce travail fait, le sociologue le diffuse dans la société. La vérification ultime de la validité de son travail, c'est que les acteurs sociaux qui prennent connaissance du travail du sociologue - ces acteurs qui produisent les nouveaux contre-arguments - vont utiliser les réductions des systèmes de conflit entre attentes sociales que le sociologue leur propose. Soit ils vont utiliser les stabilités contextuelles indiquées comme repères lointains de leurs interactions proches. Soit ils vont utiliser la révélation par le sociologue des dysfonctionnements pour tenter de construire d'autres contextes. Soit ils vont alors ne plus parvenir à raccorder leurs interactions proches à ces anciennes cohérences lointaines, mais seulement à des interactions plus proches. Le critère de véridicité de ce travail, c'est donc bien la capacité auto-réalisatrice du système de réduction et de contextualisation qui est le produit du travail sociologique, capacité auto-réalisatrice qui vérifie sa pertinence - selon les trois modes indiqués, qui vont du renforcement de la stabilité jusqu'à la déstabilisation. L'attention aux défaites, échecs et non réalisation des attentes normatives sociales est transmuée par le travail sociologique en une réduction et une diversification du 
système de réfutations croisées entre ces attentes, et cette réduction et diversification est bien, dans le meilleur des cas, auto-réalisatrice, alors même que le contenu du travail sociologique commence par partir de ce qui fait obstacle à l'auto-réalisation, et que son influence peut aussi se manifester par une déstabilisation. Mais à la différence de l'économiste, il semble difficile au sociologue de pouvoir anticiper dans quelle mesure son travail présente bien cette vertu d'auto-réalisation sociale.

Université d'Aix-Marseille I 